\title{
The Accelerator Complex from the International Design Study of the Neutrino Factory
}

\author{
F.J.P. Soler ${ }^{* \dagger}$ \\ University of Glasgow \\ E-mail: paul.soler@glasgow.ac.uk
}

The Neutrino Factory produces high-energy neutrino beams with a well-defined flavour content and energy spectrum from the decay of intense, high-energy, stored muon beams to establish CP violation in the neutrino sector. The International Design Study for the Neutrino Factory (the IDSNF) will provide a Reference Design Report (RDR) for the facility. The present baseline design has been re-evaluated to take into account the recent measurements of $\theta_{13}$. This talk describes the status of the accelerator facility and the accelerator subsystems of which it is comprised. This is a modification of the facility described in the Interim Design Report (IDR) completed in 2011. The accelerator facility will deliver $10^{21}$ muon decays per year from $10 \mathrm{GeV}$ stored muon beams. The straight sections of the storage ring point to a 100 kton Magnetised Iron Neutrino Detector (MIND) at a distance of 2000-2500 km from the source. The accelerator-physics challenges, and the R\&D underway to meet them, will be described together with alternative designs that are being developed to mitigate the technical risks that some of the subsystems present.

36th International Conference on High Energy Physics

4-11 July 2012

Melbourne, Australia

* Speaker.

${ }^{\dagger}$ On behalf of the IDS-NF Collaboration. 


\section{Introduction}

The Neutrino Factory is a new type of accelerator facility in which a neutrino beam is created from the decay of muons in flight in a storage ring [1]. The expected absolute flux and spectrum of neutrinos can be calculated with smaller systematic errors than at alternate facilities. Neutrino oscillations can be studied from the decay of both $\mu^{+}$and $\mu^{-}$thereby improving sensitivity to $\mathrm{CP}$ violation. Neutrino oscillations in matter can be extracted from the appearance of $v_{\mu}$ in far detectors (the golden channel) [2]:

$$
\begin{aligned}
& P_{v_{e} v_{\mu}\left(\bar{v}_{e} \bar{v}_{\mu}\right)}=s_{23}^{2} \sin ^{2} 2 \theta_{13}\left(\frac{\Delta_{13}}{B_{\mp}}\right)^{2} \sin \left(\frac{B_{\mp} L}{2}\right)+c_{23}^{2} \sin ^{2} 2 \theta_{12}\left(\frac{\Delta_{12}}{A}\right)^{2} \sin ^{2}\left(\frac{A L}{2}\right) \\
& +J \frac{\Delta_{12}}{A} \frac{\Delta_{13}}{B_{\mp}} \sin \left(\frac{A L}{2}\right) \sin \left(\frac{B_{\mp} L}{2}\right) \cos \left( \pm \delta_{C P}-\frac{\Delta_{13} L}{2}\right)
\end{aligned}
$$

where $\Delta_{i j} \equiv \frac{\Delta m_{i j}^{2}}{2 E_{v}}, s_{i j}=\sin \theta_{i j}, c_{i j}=\cos \theta_{i j}, J \equiv c_{13} \sin 2 \theta_{12} \sin 2 \theta_{23} \sin 2 \theta_{13}$ is the Jarlskog determinant and $B_{\mp} \equiv\left|A \mp \Delta_{13}\right|$, with $A=\sqrt{2} G_{F} n_{e}$ the matter parameter and $n_{e}$ the electron number density.

The recent discovery that $\theta_{13}$ is non-zero, $\sin ^{2} \theta_{13}=0.0241 \pm 0.0025[3,4,5,6,7]$, and global fits from all the available data $[8,9]$ suggest that the discovery of $\mathrm{CP}$ violation in the neutrino sector can be realised in future experiments. Other experimental observables that need to be determined are the neutrino mass hierarchy (the sign of $\Delta m_{31}^{2}$ ), whether $\theta_{23}$ deviates from $\pi / 4$ and the determination of the $\mathrm{CP}$ violating phase $\delta_{C P}$.

The main goal of the International Design Study for a Neutrino Factory (IDS-NF) is to provide a Reference Design Report (RDR) for a Neutrino Factory and its associated detectors by 2013. The specification of each of the accelerator, diagnostic and detector systems that make up the facility, their schedule and cost, and the physics performance of the Neutrino Factory will be delivered in the RDR. An Interim Design Report (IDR) [1] was recently published, co-sponsored by the MAP programme in the USA [10] and the EUROnu programme in Europe [11]. A schematic of the accelerator facility can be found in Figure 1.

A Magnetised Iron Neutrino Detectors (MIND) of 100 kton at a distance between 2000 and $2500 \mathrm{~km}$ shows the best performance for the discovery of CP violation [1] at the currently measured values of $\theta_{13}$ [12]. MIND is $140 \mathrm{~m}$ long and consists of large octagonal iron plates of dimensions $14 \mathrm{~m} \times 14 \mathrm{~m} \times 3 \mathrm{~cm}$, followed by two planes of scintillator bars, one with $x$ orientation and one with $y$ orientation, with each bar $1 \mathrm{~cm}$ thick and $3.5 \mathrm{~cm}$ wide (see Figure 2). A toroidal magnetic field between 1.0 and $2.2 \mathrm{~T}$ is created in the iron, by a Superconducting Transmission Line (STL) of diameter $7.8 \mathrm{~cm}$, within a $10 \mathrm{~cm}$ bore, carrying $100 \mathrm{kA}$ of excitation current. In this paper the Neutrino Factory accelerator facility will be described in more detail.

\section{Proton Driver and Target}

The proton driver includes a pulsed proton beam with average beam power of $4 \mathrm{MW}$, a repetition rate of $50 \mathrm{~Hz}$, with three bunches per train in $240 \mu \mathrm{s}, 1-3 \mathrm{~ns}$ proton bunch length and energy between 5 and $15 \mathrm{GeV}$ (with a preferred proton energy around $8 \mathrm{GeV}$ ). The beam radius should be $1.2 \mathrm{~mm}$ (RMS), with a geometric emittance less than $5 \mu \mathrm{m}$ and a $\beta^{*}$ at the target of greater than 

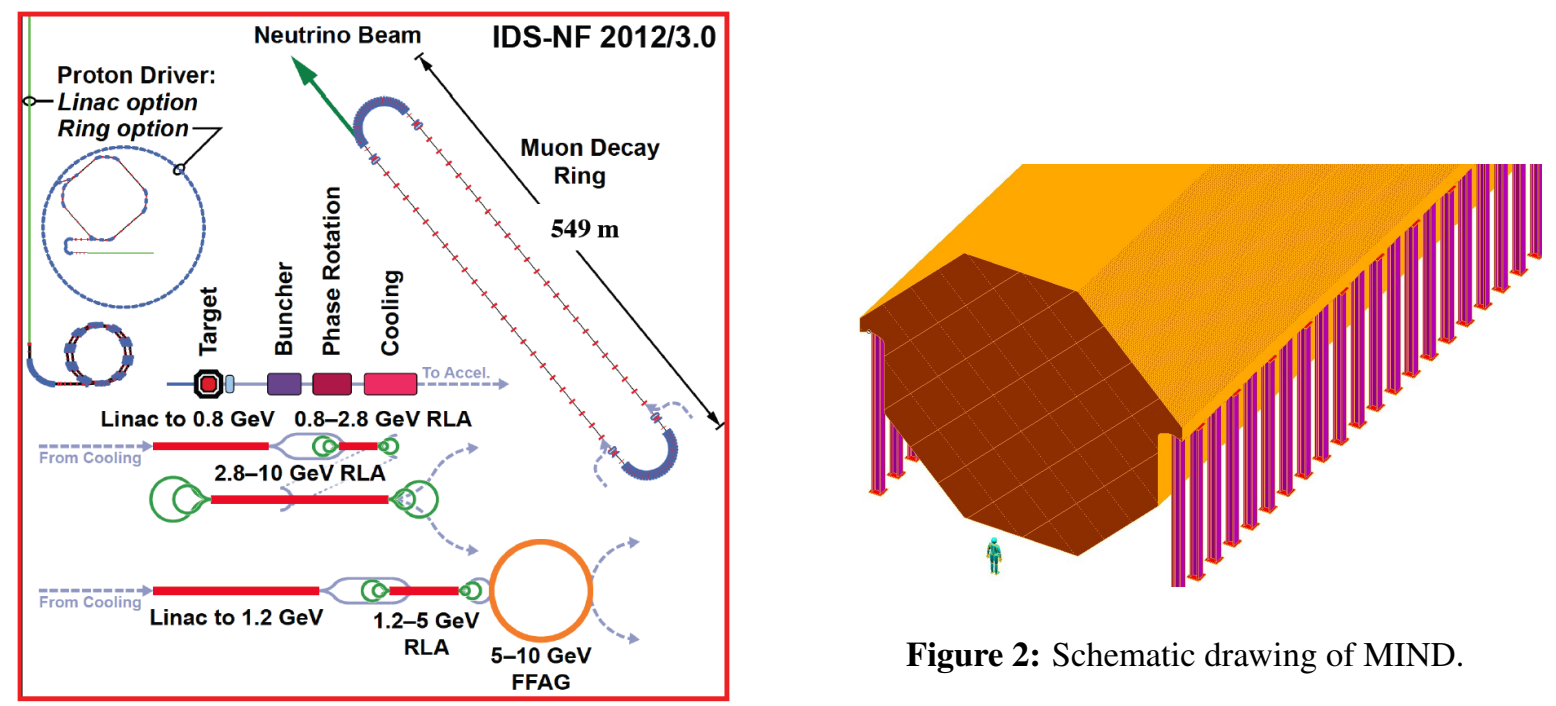

Figure 2: Schematic drawing of MIND.

Figure 1: Schematic drawing of the IDS-NF accelerator complex.

$30 \mathrm{~cm}$. Examples of proton drivers could be the Project X LINAC at Fermilab, the SPL at CERN or a Fixed Field Alternating Gradient (FFAG) at a green field site. The baseline target consists of a liquid mercury jet inside a $20 \mathrm{~T}$ capture solenoid field (Figure 3). The MERIT experiment [13] has provided a proof-of-principle of the mercury jet target.

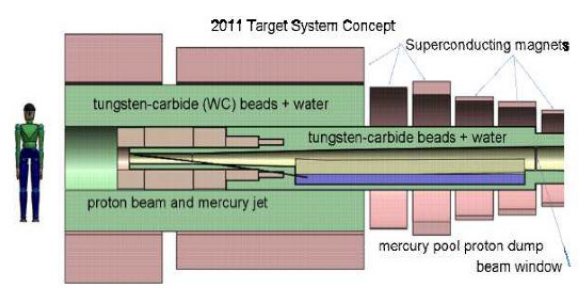

Figure 3: Neutrino Factory target.

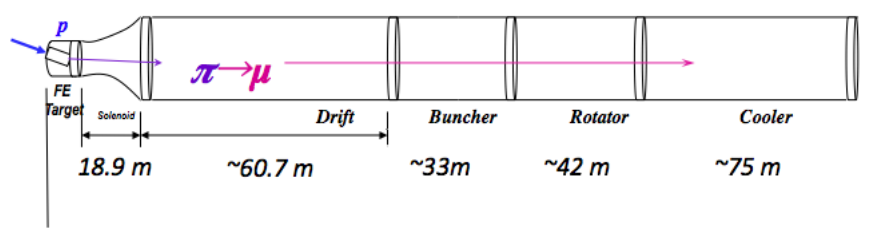

Figure 4: Neutrino Factory front-end.

\section{Muon Front End}

The Neutrino Factory muon front end consists of a pion decay channel and longitudinal drift, followed by an adiabatic buncher, phase-rotation system, and ionisation cooling channel (Figure 4). Downstream of the target solenoid, the magnetic field is adiabatically reduced from $20 \mathrm{~T}$ to $1.5 \mathrm{~T}$ over a distance of $18.9 \mathrm{~m}$. The pions then drift longitudinally over $60.7 \mathrm{~m}$ and decay to the daughter muons. The drift channel is followed by a $33 \mathrm{~m}$ buncher section that uses RF cavities of decreasing frequency (319.6 to $233.6 \mathrm{MHz}$ ) and increasing gradient (3.4 to $9.7 \mathrm{MV} / \mathrm{m}$ ) to form the muon beam into a train of bunches. Then there is a $42 \mathrm{~m}$ phase-energy rotating section that decelerates the leading high-energy bunches and accelerates the late low energy bunches, so that each bunch has the same mean energy. It consists of 56 cells of 0.75 length with normal conducting RF cavities, with frequency 230.2 to $202.3 \mathrm{MHz}$ and a gradient of $12 \mathrm{MV} / \mathrm{m}$, and superconducting coils delivering a 
$1.5 \mathrm{~T}$ focusing field. The baseline cooling channel design consists of a sequence of identical $1.5 \mathrm{~m}$ long cells, with each cell containing two $0.5 \mathrm{~m}$ long $201.25 \mathrm{MHz}$ RF cavities, two solenoid coils with opposite polarity (maximum field of $2.8 \mathrm{~T}$ providing transverse focusing with $\beta_{\perp}=0.8 \mathrm{~m}$ ), $1.1 \mathrm{~cm}$ thick $\mathrm{LiH}$ absorber discs at the ends of each cavity (four per cell) and a $0.25 \mathrm{~m}$ spacing between cavities (Figure 5). The total length of the cooling section is $75 \mathrm{~m}$ (50 cells). The cooling channel is expected to reduce the rms transverse normalised emittance from $\varepsilon_{N}=0.018 \mathrm{~m}$ to $\varepsilon_{N}=$ $0.0075 \mathrm{~m}$ (Figure 6). The Muon Ionisation Cooling Experiment (MICE) will test one cell of the muon cooling channel [14].

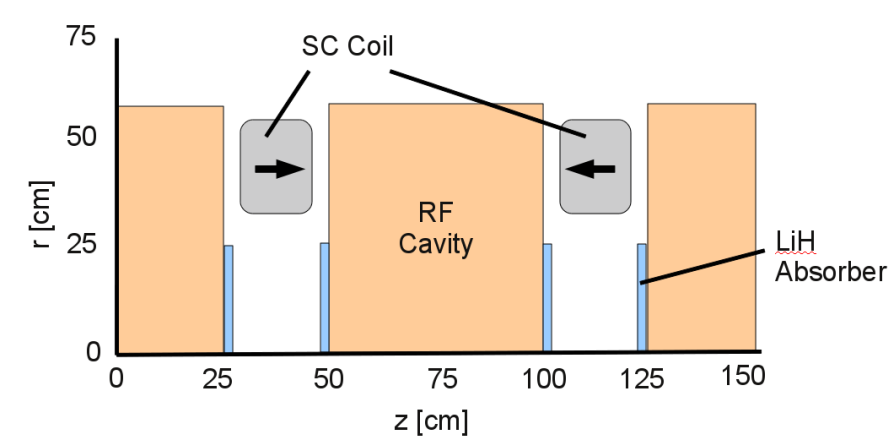

Figure 5: Baseline design of muon cooling lattice.

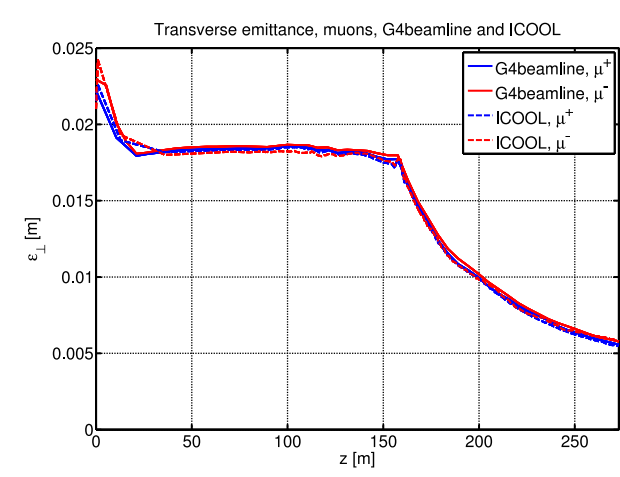

Figure 6: Cooling performance of the muon front-end.

\section{Acceleration System, Decay Ring}

The acceleration proceeds in three stages: a $146 \mathrm{~m}$ LINAC that accelerates from $0.24 \mathrm{GeV}$ to $0.8 \mathrm{GeV}$, a first Recirculating Linear Accelerator (RLA) of $79 \mathrm{~m}$ length that accelerates up to $2.8 \mathrm{GeV}$, and a second $264 \mathrm{~m}$ RLA up to $10.0 \mathrm{GeV}$ (Figure 7). There is another possibility with a LINAC up to $1.2 \mathrm{GeV}$, an RLA up to $5.0 \mathrm{GeV}$ and the final accelerating section provided by a Fixed Field Alternating Gradient (FFAG), from 5.0 to $10.0 \mathrm{GeV}$, but this option is currently disfavoured.

There is one decay ring based on a racetrack design, with long $(469 \mathrm{~m})$ straight sections followed by 15 cell arcs. The decay straights point to a far detector at a distance between 2000 and $2500 \mathrm{~km}\left(18^{\circ}\right.$ slope $)$. Muons are stored in the decay rings for about 1000 turns, with a muon beam divergence of $0.1 / \gamma$.

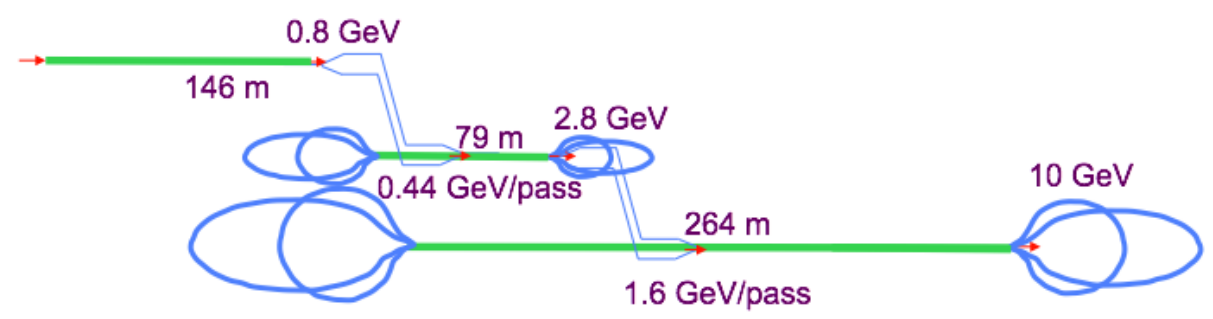

Figure 7: Accelerator system for the $10 \mathrm{GeV}$ Neutrino Factory. 


\section{Neutrino Factory Performance}

A detailed analysis of the performance of a simplified version of the detector, consisting of a square cross section $15 \mathrm{~m} \times 15 \mathrm{~m}$ immersed in a uniform dipole magnetic field of $1 \mathrm{~T}$ oriented in the positive $y$ direction, was carried out in reference [12]. The event generators NUANCE and LEPTO were used in combination with GEANT4 to simulate the neutrino deep inelastic, quasi-elastic and pion production interactions. Events were reconstructred by a Kalman filter and a comprehensive analysis was developed to select the oscillation signal, while eliminating the considerable background (see also references $[15,16]$ for further details).

At the large value of $\theta_{13}$ observed, the Neutrino Factory is optimum when the muon energy is $10 \mathrm{GeV}$, and for a single baseline with one $100 \mathrm{kton}$ MIND at a distance between 2000 and $2500 \mathrm{~km}$. The accuracy achieved in the measurement of $\delta_{C P}$ for $\theta_{13}=9^{\circ}$ is shown in Figure 8, under two assumptions of the overall normalisation and cross-section systematic errors: $\left(\sigma_{A}, \sigma_{x}\right)=$ $(3.0 \%, 2.5 \%)$ and $(1.0 \%, 1.0 \%)$. The accuracy in $\Delta \delta_{C P}$ is between $2.5^{\circ}$ and $6^{\circ}$.

These results (MIND LE) are compared with other possible facilities in Figure 9, such as LBNE, with and without Project X, the Tokai to HyperKamiokaNDE (T2HK) project in Japan, the European LBNO project with a 33 and 100 kton Liquid Argon detector, a Beta Beam with $\gamma=100$ and the MEMPHYS detector in Fréjus (BB100) and combined with a Super Beam from the CERN SPL (BB100+SPL) and, finally, not building new facilities beyond T2K and NOvA (2025). The Neutrino Factory outperforms all other facilities, with a $\delta_{C P}$ coverage between $85 \%$ and $90 \%$ for all allowed values of $\theta_{13}$.

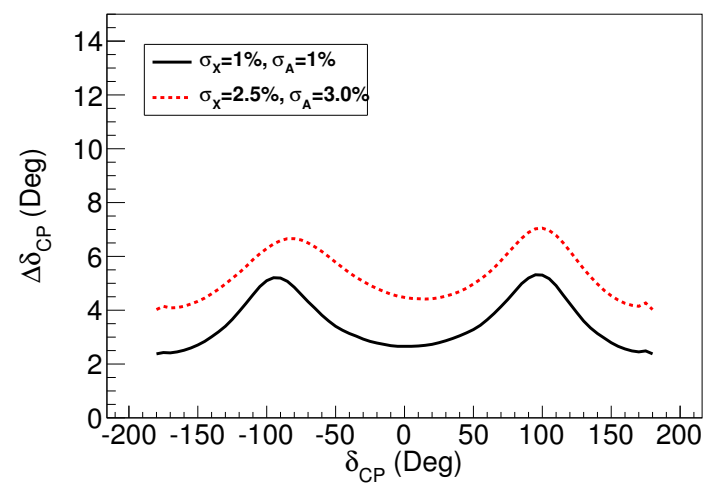

Figure 8: Precision in the value of $\delta_{C P}$ as a function of $\delta_{C P}$ under two different assumptions: top line $(3.0 \%$ normalisation and $2.5 \%$ cross-section error), bottom line ( $1 \%$ normalisation and cross-section error).

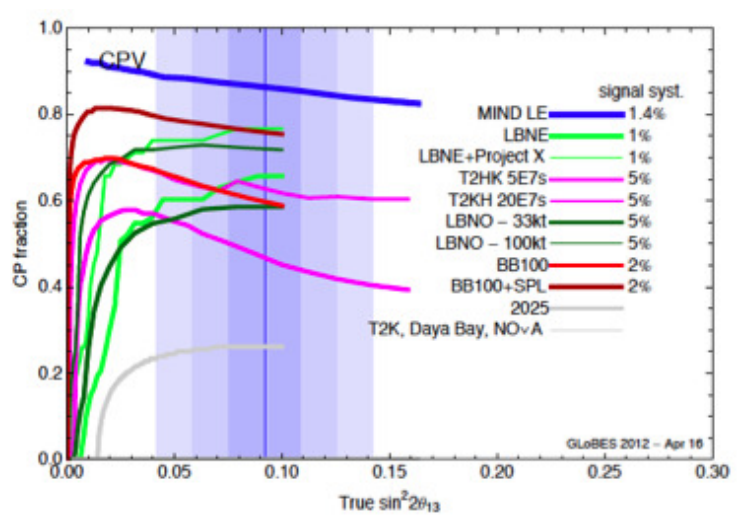

Figure 9: $\mathrm{CP}$ fraction coverage for future neutrino facilities.

\section{Conclusions}

The International Design Study for a Neutrino Factory (IDS-NF) delivered the Interim Design Report in March 2011, in which the most salient features of the accelerator facility were described. The present baseline design has been re-evaluated to take into account the recent measurements of $\theta_{13}$. The Neutrino Factory can deliver $10^{21}$ muon decays per year from a $10 \mathrm{GeV}$ stored muon 
beam, with the straight section of the storage ring pointing to a 100 kton Magnetised Iron Neutrino Detector (MIND) at a distance of 2000-2500 km from the source. The IDS-NF is on target to produce a Reference Design Report, including performance and costs, by 2013. The basic design of the facility has been achieved, including all the accelerator systems. The accuracy in the value of $\delta_{C P}$ that a Neutrino Factory can achieve and the $\delta_{C P}$ coverage by the facility is unrivalled by all other possible future facilities.

\section{References}

[1] S. Choubey, et al., International Design Study for the Neutrino Factory, Interim Design Report, IDS-NF-20, https://www.ids-nf.org/wiki/FrontPage/Documentation/IDR.

[2] A. Cervera et al., Nucl. Phys. B579, 17 (2000), arXiv:hep-ph/0002108.

[3] F. P. An et al. [Daya-Bay Collaboration], Phys. Rev. Lett. 108, 171803 (2012) [arXiv:1203.1669 [hep-ex].

[4] J. K. Ahn et al. [RENO Collaboration], Phys. Rev. Lett. 108, 191802 (2012) [arXiv:1204.0626 [hep-ex].

[5] Y. Abe et al. [Double Chooz Collaboration], Phys. Rev. Lett. 108, 131801 (2012) [arXiv:1112.6353 [hep-ex].

[6] K. Abe et al. (T2K Collaboration), Phys. Rev. Lett. 107, 041801, 2011, arXiv:1106.2822 [hep-ex].

[7] A. Holin, "New Results from the MINOS Experiment", PoS(EPS-HEP2011).

[8] G.L. Fogli, E. Lisi, A. Marrone, A. Palazzo, A.M. Rotunno, Phys. Rev. D84 053007, 2011, arXiv:1106.6028 [hep-ph].

[9] M. C. Gonzalez-Garcia, M. Maltoni, J. Salvado, T. Schwetz, JHEP12 (2012) 123, arXiv:1209.3023 [hep-ph]

[10] Muon Accelerator Programme, http://map.fnal.gov/

[11] EUROnu: A High Intensity Neutrino Oscillation Facility in Europe, http://www.euronu.org/

[12] R. Bayes, A. Laing, F. J. P. Soler, A. Cervera Villanueva, J. J. Gómez Cadenas, P. Hernández, J. Martín-Albo and J. Burguet-Castell, Phys. Rev. D 86, 093015 (2012), arXiv:1208.2735 [hep-ex].

[13] I. Efthymiopoulos "MERIT - The High Intensity Liquid Mercury Target Experiment at the CERN PS”, IEEE Nucl.Sci.Symp.Conf.Rec.1:3302-3305,2008.

[14] International Muon Ionization Cooling Experiment, http://mice.iit.edu/

[15] F.J.P. Soler, "Detectors for leptonic CP violation at the Neutrino Factory", PoS(EPS-HEP2011), 394.

[16] A. Laing, "Optimisation of detectors for the golden channel at a neutrino factory", $\mathrm{PhD}$ thesis, 2010, University of Glasgow. 